\title{
On the Observation and Simulation of Solar Coronal Twin Jets
}

\author{
Jiajia Liu ${ }^{1,3, *}$, Fang Fang ${ }^{2}$, Yuming Wang ${ }^{1,5}$, Scott W. McIntosh ${ }^{4}$, Yuhong Fan ${ }^{4}$, Quanhao Zhang ${ }^{1,3}$ \\ ${ }^{1}$ CAS Key Laboratory of Geospace Environment, School of Earth and Space Sciences, University of Science and Technology of China, Hefei, Anhui 230026, China \\ ${ }^{2}$ Laboratory For Atmospheric and Space Physics, University of Colorado at Boulder, 1234 Innovation Dr., Boulder, CO 80303, USA \\ ${ }^{3}$ Collaborative Innovation Center of Astronautical Science and Technology, Hefei 230026, China \\ ${ }^{4}$ High Altitude Observatory, National Center for Atmospheric Research, P.O. Box 3000, Boulder, CO 80307, USA. \\ ${ }^{5}$ Synergetic Innovation Center of Quantum Information \& Quantum Physics, University of Science and Technology of China, Hefei, Anhui 230026, China \\ *Corresponding Author, Contact: ljj128@ustc.edu.cn
}

\section{Contents}

\section{Introduction}

\section{Overview on Observations}

\section{Kinetics}

3.1 Kinetics of the Preceding jet . . . . . . . . . . . 3

3.2 Kinetics of the Twin jets . . . . . . . . . . .

\section{Simulation}

\section{Conclusions and Discussions}

\begin{abstract}
We present the first observation, analysis and modeling of solar coronal twin jets, which occurred after a preceding jet. Detailed analysis on the kinetics of the preceding jet reveals its blowout-jet nature, which resembles the one studied in Liu et al. 2014. However the erupting process and kinetics of the twin jets appear to be different from the preceding one. In lack of the detailed information on the magnetic fields in the twin jet region, we instead use a numerical simulation using a three-dimensional (3D) MHD model as described in Fang et al. 2014, and find that in the simulation a pair of twin jets form due to reconnection between the ambient open fields and a highly twisted sigmoidal magnetic flux which is the outcome of the further evolution of the magnetic fields following the preceding blowout jet. Based on the similarity between the synthesized and observed emission we propose this mechanism as a possible explanation for the observed twin jets. Combining our observation and simulation, we suggest that with continuous energy transport from the subsurface convection zone into the corona, solar coronal twin jets could be generated in the same fashion addressed above.
\end{abstract}

\section{Introduction}

Decades have passed since the first observations on solar jets (named as surges in Newton 1934), which are thought to play an important role in solar wind acceleration and coronal heating (e.g., Tsiropoula and Tziotziou 2004; Tian et al. 2014). A generalized definition of solar jets includes the terms of $\mathrm{H} \alpha$ surges (e.g., Canfield et al. 1996; Jibben and Canfield 2004), UV/EUV/X-ray jets (e.g., Schmieder et al. 1988; Patsourakos et al. 2008; Tian et al. 2014; Liu et al. 2015) and spicules (e.g., De Pontieu et al. 2007; Shibata et al. 2007), among which their different names come from different dominant temperatures and sizes. As shown in many previous works (Shibata et al. 1996, as a review), different jets obtain quite different physical characteristics such as length and axial speed, which range from few to hundreds megameters and ten to thousands kilometers per second, respectively.

1 Despite the different properties of different jets, it is believed that they are triggered by the similar mechanism (except type I spicules,

2 De Pontieu et al. 2007). Reconnections between newly emerging twisted loops with pre-existing ambient open fields (e.g., MorenoInsertis et al. 2008) lead to the heating and initiation of bulks of plasma, which are observed as materials of a jet (Savcheva et al. 2007). Twists transferred from the emerging flux then lead to the rotational motion of jets, as observed and studied widely in observation and simulation (e.g., Xu et al. 1984; Shibata and Uchida 1985;

5 Canfield et al. 1996; Shimojo et al. 2007; Pariat et al. 2010; Liu 2012; Liu et al. 2014; Fang et al. 2014).

Although the triggering mechanism has been studied comprehensively and thoroughly, further evolution of the system after the reconnection and the detailed energy budget during jet events still stays unclear. As known by the community, during a jet event, the magnetic free energy is released through two ways. One is reconnection and the other is post-reconnection relaxation of the magnetic field structure, which is always manifested by the rotational motion of a jet. Observation employing the unprecedented combination of the SDO (Pesnell et al. 2012) and STEREO (Kaiser et al. 2008) data of a solar EUV jet by Liu et al. (2014) has demonstrated that the continuous relaxation of the post-reconnection magnetic field structure is an important energy source for a jet to climb up higher than it could through only reconnection. Analysis in Liu et al. (2014) shows that the kinetic energy of the jet gained through the relaxation is about 1.6 times of that gained from the reconnection. The importance of the post-reconnection relaxation process which introduces upward Lorentz force has also been demonstrated in the 3D MHD numerical simulation work by Fang et al. (2014).

Further releasing of magnetic free energy may take place in terms of recurring jets. When persistent flow continuously injects energy into the corona from the sub-surface regions, recurring jets may present (Pariat et al. 2010). However, in this paper, we will present the observation and analysis on another possibility - solar coronal twin jets after a preceding blowout jet. After the detailed analysis on the kinetics, we continue the 3D MHD simulation work in Fang et al. (2014) where a sub-photospheric buoyant magnetic flux rope emerges into the corona and reconnects with the ambient fields, producing a blowout jet, and demonstrate that the twin jets are generated via the reconnection between the ambient open fields with a highly twisted sigmoidal magnetic flux which is the outcome of the evolution of the magnetic field configuration during the preceding jet. Based on the observations in Section 2 and 3, and the simulation in Section 4, we present our conclusions and discussions in the 
last section

\section{Overview on Observations}

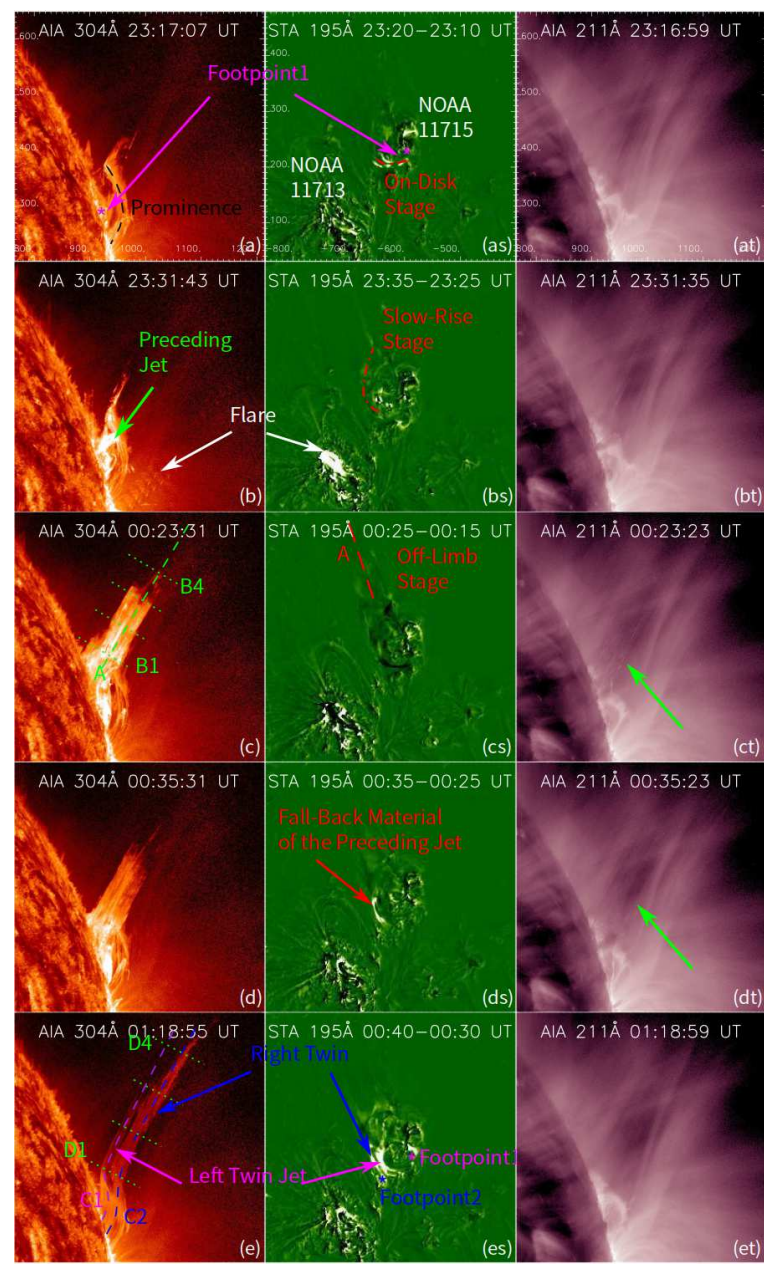

Figure 1: Time-sequential observations on the analyzed event. (a) and (as): simultaneous observation on the on-disk stage of the preceding jet by SDO and STEREO. The purple asterisk shows the footpoint region ("Footpoint1") of the preceding jet. The prominence is found not to interact with the jets and kept isolated from them. (b) and (bs): observation on the slow-rise stage of the preceding jet. (c) and (cs): observation when the preceding jet reached its maximum height. Slice "A" is placed along the jet's axis to help analyze its axial motion, with slices "B1" to "B4" perpendicular to the axis. (d) and (ds): one moment during the descending phase of the preceding jet. (e) and (es): observation of SDO when the twin jets reached their maximum heights and of STEREO shortly after the twins were triggered. Slices "C1" and "C2" are placed along the jets' axes, with slices "D1" to "D4" perpendicular to the jets' axes. (at)-(et): simultaneous AIA $211 \AA$ A observations with the green arrows indicating the hot component of the preceding jet.

The event focused on in this paper started around the midnight between April $10^{\text {th }}$ and the following day in the year 2013, performed an attractive show and ended after its 3-hour performance on the north-west limb of the Sun in the field-of-view (FOV) of the Solar Dynamics Observatory (SDO)/AIA. A solar coronal jet surfed high into the solar corona before falling back to the solar surface. And another two jets came out around the end of the off-limb stage of it. The first jet could be found in all seven UV/EUV channels of SDO/AIA and in EUV observations by the Solar TErrestrial RElations Observatory (STEREO)/EUVI, indicating the multi-thermal nature with a wide temperature range at least from ten thousands to ten millions in units of Kelvin (See online animation M1) (Lemen et al. 2012). However, as we can see from the $211 \AA$ observations in Figure 1, there were only few jet materials resolved and the jet was dominated by warm materials at the temperature of $304 \AA$ passband. Running-difference images in animation M1 reveal that materials of the jets in different temperatures acted in the same way. A prominence could also be found at almost the same position seen from the $S D O$ during the analyzed event (Figure 1(a)). However, as it is found to remain its main structure through out the whole event and not show any obvious interaction with the jets, we will not take it into account during our analysis in this paper.

The first jet, which will be referred to as the "preceding jet" hereafter, originated from a footpoint region, says "Footpoint1", located around $\mathrm{E} 38^{\circ} \mathrm{N} 18^{\circ}$ within the active region NOAA 11715 in the FOV of STEREO-A (Figure1(as)). This location corresponded to $\mathrm{W} 95^{\circ}$ from the point of view of $S D O$ taking the separation between these two probers of $133^{\circ}$ into account, which means that some early (on-disk) evolution of the jet would hide from $S D O$ observations - consistent with our temporal investigations on animation M1. The preceding jet was triggered around 23:17 UT April 10 ${ }^{\text {th }}$, and continuous chromospheric activities could be found around its footpoint region within the same active region before its excitation via STEREO observations. The jet traveled on disk for about 42 Mm until 23:20 UT (red dashed curve in Figure 1(as)). It could only be seen as a small bright region during this on-disk stage from the $S D O$, shown as a purple asterisk in Figure 1(a) (and animation M2). Then, the jet was found to ascend slowly (slow-rise stage) to a height of $26 \mathrm{Mm}$ in SDO observation (Figure 1(b)) and simultaneously continued its on-disk motion traveling for about $137 \mathrm{Mm}$ in STEREO observation (shown as the red dashed curve Figure 1(bs)) before 23:32 UT. Additionally, a C3.9 flare could also be observed in the neighboring active region NOAA 11713 (Figure 1(as)), which started at around 23:31 UT (Figure 1(b) and (bs)) during the jet's slow-rise stage. However, we'll not talk much into the relation between the jet and the flare as they were not in the same active region and their relations might be complicated which is beyond the topic of this paper.

After this slow-rise stage, the preceding jet began to ascend almost straightly off the limb with an average width of about $38 \mathrm{Mm}$ (off-limb stage, Figure 1(c) and (cs)). The jet reached its maximum height at around 00:23 UT April $11^{\text {th }}$ (Figure 1(c)) and started to fall back to the solar surface after that. The falling-back materials could also be found in STEREO observations (Figure 1 (ds)). Similar as the one studied in Liu et al. (2014), rotational motion of the jet materials could be found over the whole off-limb stage of the jet.

Around the end of the off-limb stage of the preceding jet (00:3500:40 UT), another two jets were triggered. They stayed so close in the FOV of $S D O$ that they could be easily mistaken for one jet. However as we can see from STEREO observations, the left one originated from a footpoint region that was apparently around that of the preceding one, which located inside the active region NOAA 11715 ("Footpoint1", Figure 1(es)). While the right one was found to be originated from another footpoint region ("Footpoint2", Figure 1 (es)), which was south of "Footpoint1" and between the two active regions NOAA 11715 and 11713. Meanwhile, there was a 
gap between them with the distance of their axes about $20 \mathrm{Mm}$ and widths of their tunnels about $14 \mathrm{Mm}$ and $18 \mathrm{Mm}$ respectively in SDO observations. Moreover, they finally reached different heights.

On the other hand, they are considered as "twin jets" rather than "two jets" due to: (1) they were simultaneously triggered with close footpoints and (2) they stayed very close together and shared plenty of commonalities in temperature, brightness and kinetic properties, as we will see in the rest of this paper. No obvious flux emergence could be found around the twins' footpoint regions through the observations of STEREO during their initiation. Meanwhile, only transient rotational motion of the twins could be found during their early off-limb stage. The rotational motion appeared to be much weaker than that of the preceding jet, with longer periods and much shorter lifetime. No rotational motion could be found thereafter.

\section{Kinetics}

One major concern when analyzing the kinetics of the observed jets is the projection effect, which may have pretty large influence on the resolved axial velocity of the materials within the jets. To correct the off-limb projection effect, we combine the observations from the two satellites (SDO and STEREO-A) employing a simple trigonometric analysis as done in Liu et al. (2014). Knowing the separation of SDO and STEREO-A of about $133^{\circ}$, the projected length of the preceding jet at 00:23 UT of about 145 Mm in the view of $S D O$ (Figure 1(c)) and $98 \mathrm{Mm}$ at almost the same time in the view of STEREO (Figure 1(cs)), we find that the axis of the preceding jet was about $62^{\circ}$ inward the plane of the sky in the view of $S D O$. The actual (physical) off-limb length and the axial speeds of the jet should be corrected with a factor of $\cos ^{-1}\left(62^{\circ}\right) \approx 2.11$.

\subsection{Kinetics of the Preceding jet}

To investigate the kinetics of the preceding jet during its offlimb stage, we place a slice "A" along its axis as shown in Figure 1(c) with a width $38 \mathrm{Mm}$ (same as that of the jet). The runningdifference time-distance diagram derived from slice " $A$ " is shown in Figure 2(A).

Sub-jets expelled successively as parts of the preceding jet could be found as bright-dark alternating stripes in Figure 2(A). As indicated by the black arrow, materials before the preceding jet were not sub-jets of the preceding jet - they were ejactas from the prominence labeled by the black dashed line in Figure 1(a). Tracing the trajectories of several sub-jets in the time-distance plot to investigate the very detailed kinetics of them (as done in Liu et al. 2014) turns out to be impossible for this particular event, due to the poor contraries between these sub-jets and their intermissions.

These sub-jets turned out to have an upward acceleration below a certain height and then started to decelerate. The exact value of the acceleration of the sub-jets near the bottom can not be well estimated, due to the bad contraries and that different sub-jets seemed to yield different values. However, the downward acceleration of these sub-jets during the late ascending phase turned out to be similar with that during the descending phase. Blue stars in Figure 2(A) shows the trajectory of one of the sub-jets, which can be well fitted by a parabolic function, shown as the blue dashed curve in Figure 2(A). The downward acceleration obtained by the parabolic fit is about $-181.8 \pm 9.6 \mathrm{~m} \mathrm{~s}^{-2}$. We can conclude that similar as the one studied in Liu et al. (2014), sub-jets of the preceding jet experienced an "accelerating-decelerating-falling" process during its

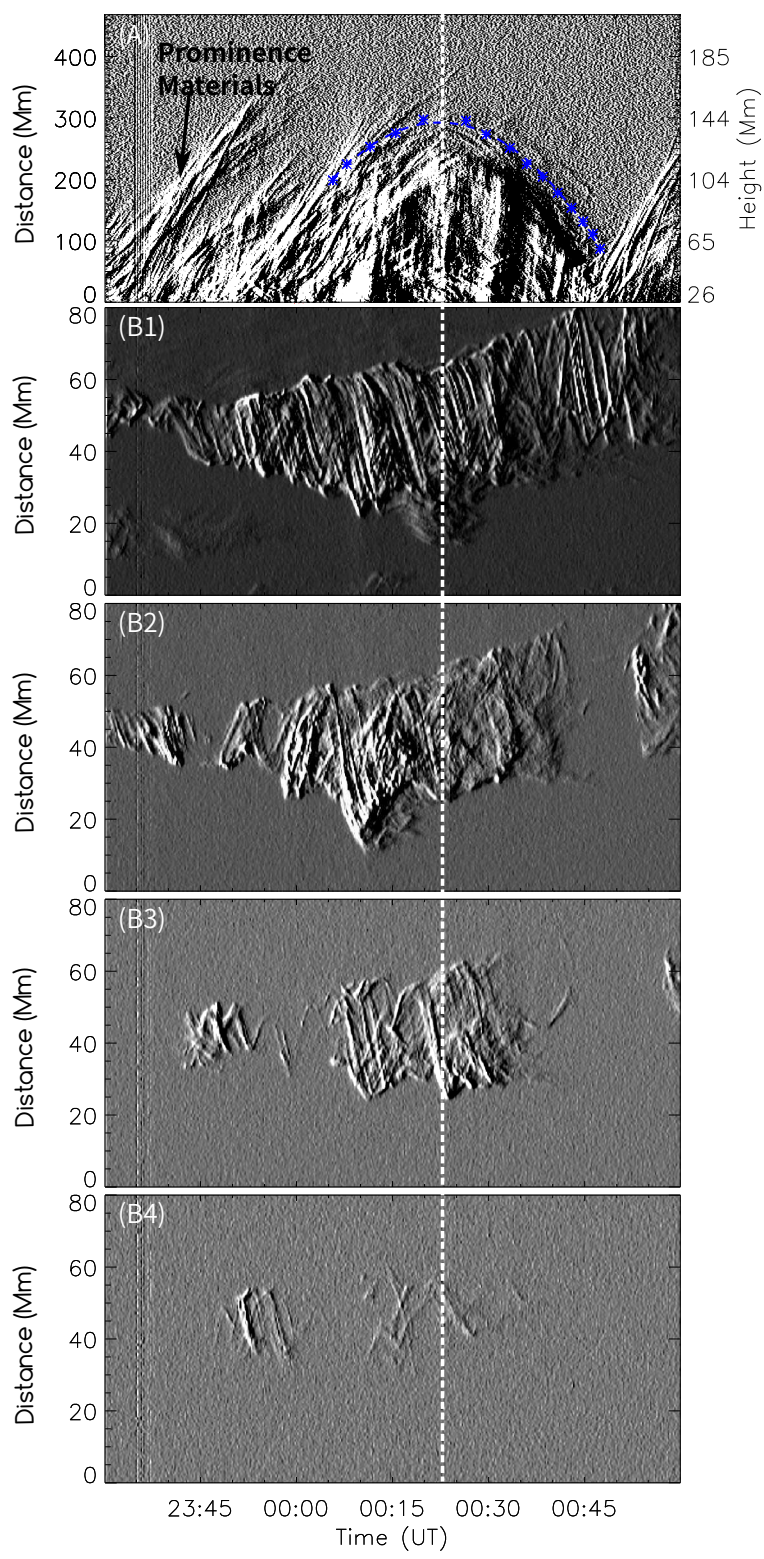

Figure 2: (A): running-difference time-distance plot of slice "A" in Fig 1(c) with the left y-axis distance along the slice after correcting the projection effect and the right $y$-axis height relative to the solar limb. The blue stars show one of the most distinguished tracks of the resolved sub-jets within the preceding jet, with the blue curve its parabolic fitting result. The black arrow indicates materials ejected from the prominence labeled in Fig 1(a). (B1)(B4): running-difference time-distance plots of slice "B1" to "B4" in Fig 1(c). Continuous rotational motion could be found in all the four panels with roughly the same period. The vertical dashed white line shows the time when the jet reached its maximum height.

off-limb stage. And the downward acceleration during the late ascending phase and whole descending phase are both less then the average gravity $\left(-220 \mathrm{~m} \mathrm{~s}^{-2}\right)$.

Continuous rotational motion could also be observed during the whole off-limb stage of the preceding jet. Four parallel slices named "B1" to "B4" are placed at four different distances (36 Mm, $126 \mathrm{Mm}, 216 \mathrm{Mm}$ and $306 \mathrm{Mm}$ ) perpendicular to the jet's axis to 
help probe the rotational motion (Fig 1(c)). All the four slices are oriented toward lower latitudes. The resulting running-difference time-distance plots of them are shown in Figure 2(B1) to (B4). Besides the expanding (Figure 2(B1)) and swinging-toward-lowerlatitude (Figure 2(B1)-(B3)) motion of the jet, bundles of threads rotating around the jet's axis could also be observed easily via the sine-like tracks in the time-distance plots. Although there are not strong signal of opposite propagating motion for some threads in Figure 2(B1) due to shadowing effect of optically thick materials, turning motion of them around the edges, continuous investigation from (B2) to (B4) and animation M2 together can indicate us their rotational motion. As seen from the diagrams, the rotational motion kept almost the same period at these four different heights and didn't show obvious deceleration after the jet reaching the maximum height (the white dashed line indicates the time when the jet reached the maximum height).

Analysis employing a sine-function fit along these sine-like tracks on the four time-distance plots reveals the periods of the rotational motions at these four different heights of $564 \pm 79 \mathrm{~s}$, $586 \pm 46 s, 557 \pm 147 s$ and $606 \pm 35 s$ respectively. These similar values again support our visual observation that the rotational motion kept almost the same behavior at different heights and didn't apparently slow down with time. As the last slice gives only few tracks that may lead to large errors, we average the periods obtained from the first three slices to estimate the average period of the rotational motion, which turns out to be $569 \mathrm{~s}$ (with an error 91 s). Taking the average width $38 \mathrm{Mm}$ of the jet into account, the average line speed of the rotational motion during the off-limb stage is then estimated to be about $209.8 \pm 33.6 \mathrm{~km} \mathrm{~s}^{-1}$. As the upward Lorentz force acting on the jet after reconnection was resulted from the untwisting motion (Shibata and Uchida 1985), the nearly invariable rotating period then is consistent with the above result that the downward acceleration in the late ascending phase and in the descending phase were almost the same.

\subsection{Kinetics of the Twin jets}

Similar method reveals almost the same inclination of the twins' axes as the preceding jet related to the plane of the sky. To probe the axial motions of the twin jets, we place two slices along their axes labeled as "C1" and "C2" in Figure 1(e) for the left and right twin jet respectively. Their corresponding running-difference timedistance plots are shown in Figure 3(C1) and (C2) with the y-axis the distance along the slices after correcting the projection effects.

As in Figure 3(C1), the left twin jet reached a maximum distance along the slice of about 330 Mm at around 01:09 UT. The downward acceleration during its ascending phase and descending phase turned out to be similar. Parabolic fitting (blue dashed curve in Fig. 3(C1)) on one of the trajectories (blue stars in Fig. 3(C1)) gives us an average deceleration of about $-228.9 \pm 8.1 \mathrm{~m} \mathrm{~s}^{-2}$. It is hard to determine the maximum distance of the right twin jet (Fig. 3(C2)) it seemed to reach a distance that was beyond the FOV of SDO observations or faint to be invisible. However the dynamics seems to be similar as the left one with the average downward acceleration of about $-234.5 \pm 10.7 \mathrm{~m} \mathrm{~s}^{-2}$ (one of the trajectories is shown as the blue stars with the parabolic fitting result the blue dashed curve in Fig. 3(C2)). All of the above values resemble the average local gravity $\left(-220 \mathrm{~m} \mathrm{~s}^{-2}\right)$, which indicates that there was almost no extra driving force acting on the twins.

Meanwhile, we place four parallel slices "D1" to "D4" at different heights perpendicular to the twins' axes to investigate the rotational motion of the twins if there was any. Corresponding running-
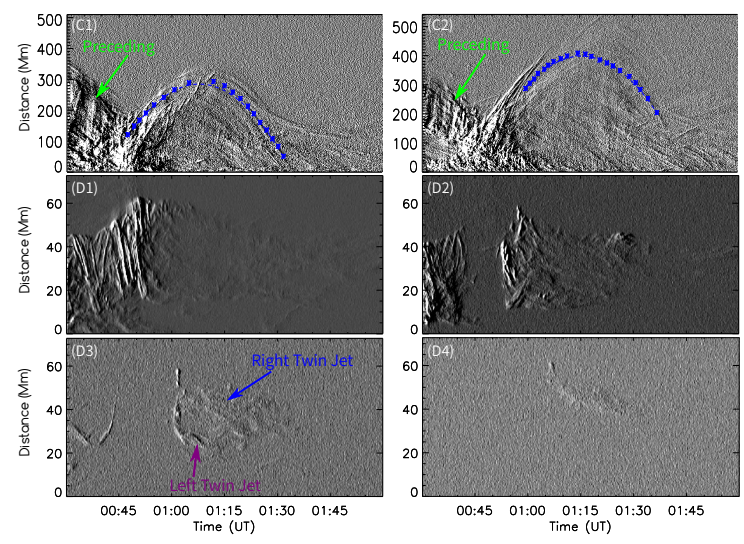

Figure 3: (C1) and (C2): running-difference time-distance plots for slice "C1" and "C2" in Fig. 1(e) along the axis of the left and right twin jet respectively. The blue stars represent trajectories of materials within the twins, with the blue curves their parabolic fitting results. (D1) to (D4): running-difference time-distance plots for slices "D1" to "D4" perpendicular to the twins' axes as shown in Fig. 1(e). Only very weak rotational motion of the twins could be found at their early off-limb stage from these four time-distance plots.

difference time-distance plots are shown in Figure 3(D1) to (D4) with the y-axis the distance along the slices from higher latitudes. It is hard to find the twins in panel (D1) because the preceding jet was much brighter than the twins. Very weak rotational motions with very few turns, long period, low velocity and small amplitude of the twins during their early ascending phase could be found in panel (D2). However, it is hard to distinguish them from each other in this panel because they were too close together. The twins became more distinct from each other in panel (D3) and the left twin jet disappeared in panel D4 because slice D4 is located at a height beyond the left twin jet could reach. Then, we can conclude from these four panels that the twins only showed very weak rotational motion during their early off-limb stages, which is very different from the preceding one.

\section{Simulation}

Fang et al. (2014) studied the eruption of coronal jets by numerical simulations on the interaction between emerging and preexisting magnetic fields. A stationary central-buoyant twisted magnetic flux rope was initially imposed in the convection zone just underneath the photosphere, and it started to emerge immediately under magnetic buoyancy. The emergence of a subsurface magnetic structure in such a stratified atmosphere from the convection zone into the corona gives rise to dramatic expansion of the emerging fields. Due to the initial setup of the directions of the emerging and ambient fields, the emerging fields in the outer periphery of the flux rope reconnect immediately with the ambient open fields, producing a thin column of plasma outflow, identified as "standard jet", following the categorization by Moore et al. (2010). Further reconnection also opens up the overlying confining field for the emerging core in the flux rope, and promotes the further emergence of the flux rope. In addition, strong current builds up within the flux rope and reconnection takes place underneath the core field, releasing the twisted core into the corona. The reconnection between 
the core field and the open ambient field drives a violent eruption in the corona, generating a much wider column of plasma outflow, known as "blowout jets", as shown by previous simulations (Archontis and Hood 2013; Moreno-Insertis and Galsgaard 2013). In particular, the outward motion of the ejected plasma in the jet is accompanied with an apparent rotating motion (Tian et al. 2011). Comparison of the magnetic twist, upward motion and rotational motion shows that along magnetic field lines with high twist, the plasma moves upward with a simultaneous spinning motion (Pariat et al. 2009). Further analysis of the Poynting energy flux shows that it is the Poynting flux that drives magnetic energy outward together with mass flow driven by Lorentz force and propagation of magnetic twist.

The characteristics of the preceding jet observed in this paper fit most of the theoretical/numerical results described above and could be easily identified as a blowout jet. However, the identity of the following twin jets and their triggering mechanism seem to be much more complicated and unreadable. First of all, no obvious falling-back materials of the preceding jet have been detected from the investigation on the STEREO observations (online animation M1 and Figure 1(ds)) at the location of the footpoint region of the right twin jet ("Footpoint2" in Figure 1(es)) before its initiation, which indicates that the twins should not be triggered by the falling-back materials of the preceding one. Secondly, there was not any apparent chromospheric activity detected before the initiation of the twin jets, excluding the possibility of simple standard/blowout jets which were triggered by flux emergence or underneath shearing/rotational motions. Finally, the twins acted totally simultaneously when they were triggered, stayed very close together during their eruption and obtained similar kinetic parameters, which inspire us that they should have the same triggering mechanism. Due to the lack of underneath magnetic field observations and poor spacial/temporal resolutions of the STEREO EUVI instruments, we are not able to analyze the exact triggering mechanism of the twins directly from the observations.

To investigate the possible mechanisms that trigger the twin jets, we continue the simulation in Fang et al. (2014) after the eruption of blowout jet. During the blowout jet, sigmoidal current sheet forms in the emerging flux rope, together with the inverse-S shaped magnetic field configuration, shown in Figure 4(a). The reconnected field lines consist of elongated sigmoidal magnetic fields embedded in the open fields. The distribution of the current density $|J| /|B|$ represented by the color of the rods clearly shows that the sigmoidal magnetic fields are loaded with strong current in the two ends outlined by the two dashed rectangles, as well as the center. We also note that the directions of the sigmoidal fields are aligned in the $+\mathrm{Y}$ direction in the dashed rectangles at the two ends of the sigmoid, which are anti-parallel to the ambient fields in the -Y direction. The configuration of the magnetic fields and the current in these two regions then give rise to reconnection between the sigmoidal and ambient fields at the two ends, driving mass outflow along the field lines at the two arms of the sigmoidal structure, accompanied with energy flux. As shown by the evolution of total energy flux at the photosphere in Figure 4(b), there is a persistent energy flow into the corona from the subsurface convection zone during the simulation. To further study the observational effect of the reconnection at the two sites, we calculate the synthetic emission from the simulation data and observe the structure from multiple points of view, as shown in Figure 5. Panel (a) shows an X-Z plane view of data, which is perpendicular to the direction of the original flux rope. Here two dome structures form in the lower corona, each corresponding to one of the two arms of the sigmoidal
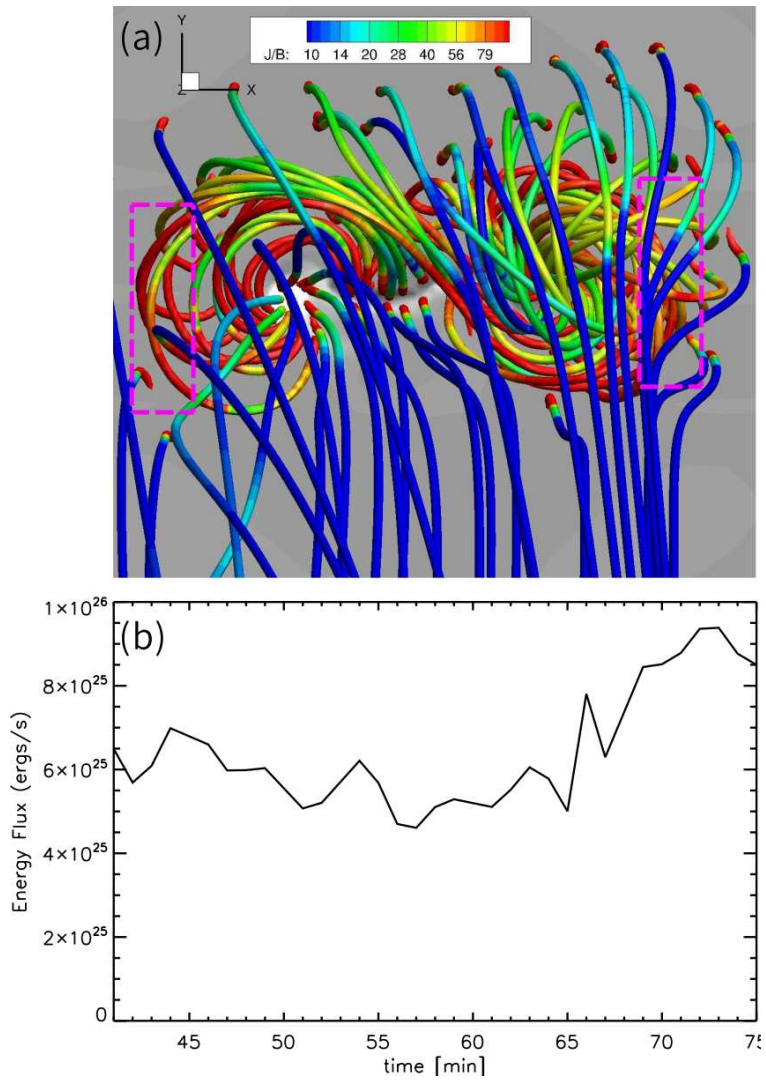

Figure 4: (a): 3D structure of the magnetic fields in the simulation around the end of the preceding blowout jet at the time of $60 \mathrm{~min}$. The grey plane shows the photospheric magnetogram and the rods represents the field lines with color showing the $|J| /|B|$ in units of $\mu A m^{-2} G^{-1}$. (b): the evolution of total energy flux at the photosphere.

fields, with one bright column of jet with outflowing plasma extending from each dome. Panel (b) is viewed from Y-Z plane, parallel to the axis of the original flux rope, and the two simultaneous jets are easily identified here. Panel (c) shows a top view of the domain, with a bright sigmoidal shape structure. At the two arms of the sigmoid, there is a significant increase of brightness in the emission. The brightening at the two arms results from the reconnection of the sigmoidal fields with the ambient fields at the two ends, producing two simultaneous jets with energy and mass outflows as shown in Panel (a) and (b). Panel (d) gives the observed twin jets in the $304 \AA$ passband of the AIA, which is compared favorably with the simulation in Panel (b). A temporal picture of Figure 5 showing the entire simulated evolving process could be found in animation M3.

Following the investigation in the simulation results, we propose that the twin jets observed here are probably generated in the same fashion, i.e., via the reconnection of ambient fields and a highly twisted sigmoidal magnetic structure, which might result from the reconnection during the blowout jets.

\section{Conclusions and Discussions}

In this paper, we presented the first observation and simulation on solar coronal twin jets after a preceding solar coronal jet. As to the preceding jet, observations and analysis indicate its blowout jet 

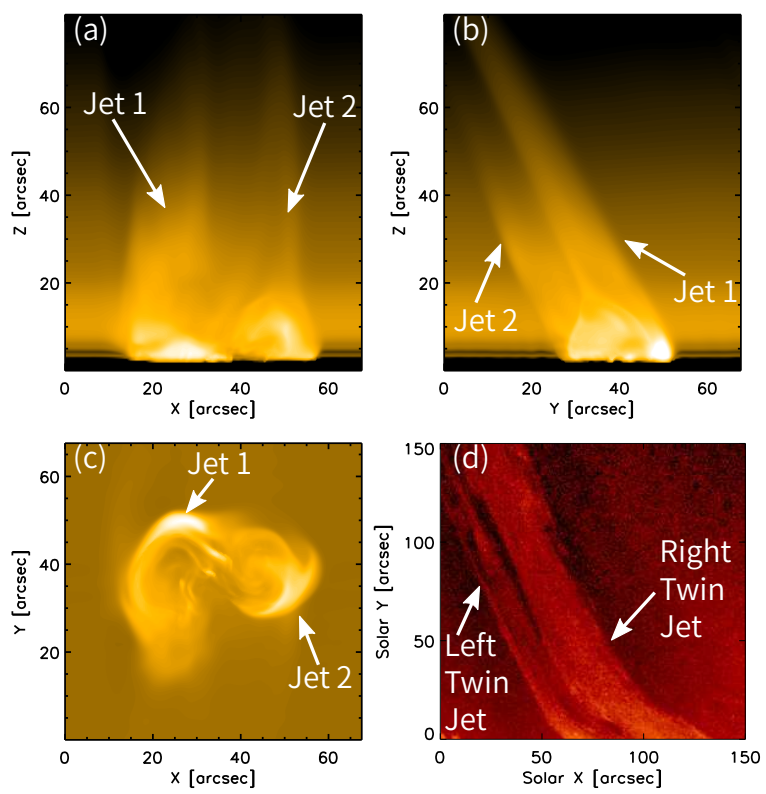

Figure 5: Synthetic emission in $171 \AA$ from the simulation at the time of $75 \mathrm{~min}$ as viewed from XZ (a), YZ (b) and XY (c) plane. An online move (M3) shows the detailed simulated evolution of the twin jets as viewed from these three planes. Panel (d) gives the observed twin jets in the $304 \AA$ passband of the AIA, the image has been rotated $50^{\circ}$ counter-clockwise.

nature.

Like the jet analyzed in Liu et al. (2014), the preceding jet underwent an acceleration during its on-disk, slow-rise and early off-limb stages, which is widely believed to be majorly introduced by the magnetic reconnections between emerging flux and ambient open fields. After then, the jet kept rising under the Lorentz force working. Continuous rotational motion of the jet's material around its axis could also be observed during the whole off-limb stage. Sinefunction fit shows that the rotational motion kept almost the same period of about $569 \pm 91 \mathrm{~s}$ at different height and did not changed much with time.

Significantly different from the preceding blowout jet, without apparent underneath activities before their initiations, the twin jets were triggered around the end of the off-limb stage of the preceding one. They were shot out with pretty high axial speeds but rare rotational motions. In lack of the detailed information on the magnetic fields in the twin jet region, we instead use a numerical simulation using a 3D MHD model as described in Fang et al. (2014), and find that in the simulation a pair of twin jets form due to reconnection between the ambient open fields and a highly twisted sigmoidal magnetic flux which is the outcome of the further evolution of the magnetic fields following the preceding blowout jet. Based on the similarity between the synthesized and observed emission we propose this mechanism as a possible explanation for the observed twin jets.

Precise estimation and comparison on the energy budgets of the preceding and twin jets would hint us more details of the realistic magnetic field configuration and mechanism. However, as we could not determine the mass of the jets and work done by forces accurately, this comparison is not able to be done for now. Detailed analysis on the energies from simulation results in the future may shed light on this issue. On the other hand, the sigmoidal struc- ture, which was a remarkable signature during the twin jets event according to the simulation, was not observed directly in this paper probably due to the relatively low cadence and resolution of the STEREO/EUVI instruments and/or different temperatures. More work in exploring and analyzing such twin jets in the future would promisingly lead to improvements.

Acknowledgments. We acknowledge the use of data from AIA instrument on board Solar Dynamics Observatory (SDO), EUVI instrument on board Solar TErrestrial RElations Observatory (STEREO). This work is supported by grants from China Postdoctoral Science Foundation, MOST 973 key project (2011CB811403), CAS (Key Research Program KZZDEW-01-4), NSFC (41131065 and 41121003), MOEC (20113402110001) and the fundamental research funds for the central universities. J.L did part of this work when he was a student visitor at HAO and supported by the Chinese Scholarship Council (201306340034). F.F is supported by NASA Grant NNX13AJ04A and the University of Colorados George Ellery Hale Postdoctoral Fellowship.

\section{References}

Archontis, V., and A. W. Hood, A Numerical Model of Standard to Blowout Jets, ApJL, 769, L21, doi:10.1088/2041-8205/769/2/L21, 2013.

Canfield, R. C., K. P. Reardon, K. D. Leka, K. Shibata, T. Yokoyama, and M. Shimojo, H $\alpha$ Surges and X-ray Jets in AR 7260, ApJ, 464, 10161029,1996

De Pontieu, B., S. McIntosh, V. H. Hansteen, M. Carlsson, C. J. Schrijver, T. D. Tarbell, A. M. Title, R. A. Shine, Y. Suematsu, S. Tsuneta, Y. Katsukawa, K. Ichimoto, T. Shimizu, and S. Nagata, A Tale of Two Spicules: The Impact of Spicules on the Magnetic Chromosphere, PASJ, 59, 655, 2007.

Fang, F., Y. Fan, and S. W. McIntosh, Rotating Solar Jets in Simulations of Flux Emergence with Thermal Conduction, ApJL, 789, L19, doi:10. 1088/2041-8205/789/1/L19, 2014.

Jibben, P., and R. C. Canfield, Twist Propagation in $\mathrm{H} \alpha$ Surges, ApJ, 610(2), $1129-1135,2004$

Kaiser, M. L., T. A. Kucera, J. M. Davila, O. C. St. Cyr, M. Guhathakurta, and E. Christian, The STEREO Mission: An Introduction, SSR, 136, 516, doi:10.1007/s11214-007-9277-0, 2008.

Lemen, J., A. Title, D. Akin, P. Boerner, C. Chou, J. Drake, D. Duncan, C. Edwards, F. Friedlaender, G. Heyman, N. Hurlburt, N. Katz, G. Kushner, M. Levay, R. Lindgren, D. Mathur, E. McFeaters, S. Mitchell, R. Rehse, C. Schrijver, L. Springer, R. Stern, T. Tarbell, J.-P. Wuelser, C. Wolfson, C. Yanari, J. Bookbinder, P. Cheimets, D. Caldwell, E. Deluca, R. Gates, L. Golub, S. Park, W. Podgorski, R. Bush, P. Scherrer, M. Gummin, P. Smith, G. Auker, P. Jerram, P. Pool, R. Soufli, D. Windt, S. Beardsley, M. Clapp, J. Lang, and N. Waltham, The atmospheric imaging assembly (aia) on the solar dynamics observatory (sdo), Solar Physics, 275(1-2), 17-40, 2012.

Liu, J., Y. Wang, R. Liu, Q. Zhang, K. Liu, C. Shen, and S. Wang, When and how does a Prominence-like Jet Gain Kinetic Energy?, ApJ, 782, 94, 2014.

Liu, J., Y. Wang, C. Shen, K. Liu, Z. Pan, and S. Wang, A Solar Coronal Jet Event Triggers a Coronal Mass Ejection, ApJ, 813, 115, 2015.

Liu, W., Period doubling in magnetospheric convection cycle, Journal of Geophysical Research, 117(A4), 1-8, doi:10.1029/2011JA017090, 2012.

Moore, R. L., J. W. Cirtain, A. C. Sterling, and D. A. Falconer, Dichotomy of Solar Coronal Jets: Standard Jets and Blowout Jets, ApJ, 720, 757770, doi:10.1088/0004-637X/720/1/757, 2010.

Moreno-Insertis, F., and K. Galsgaard, Plasma Jets and Eruptions in Solar Coronal Holes: A Three-dimensional Flux Emergence Experiment, ApJ, 771, 20, doi:10.1088/0004-637X/771/1/20, 2013. 
Moreno-Insertis, F., K. Galsgaard, and I. Ugarte-Urra, Jets in Coronal Holes: Hinode Observations and Three-dimensional Computer Modeling, ApJL, 673, L211-L214, 2008.

Newton, H. W., The Distribution of Radial Velocities of Dark H $\alpha$ Markings near Sunspots, MNRAS, 94, 472, 1934.

Pariat, E., S. K. Antiochos, and C. R. DeVore, A Model for Solar Polar Jets, ApJ, 691, 61-74, doi:10.1088/0004-637X/691/1/61, 2009.

Pariat, E., S. K. Antiochos, and C. R. DeVore, Three-dimensional modeling of quasi-homologous solar jets, The Astrophysical Journal, 714(2), 1762, 2010.

Patsourakos, S., E. Pariat, A. Vourlidas, S. K. Antiochos, and J. P. Wuelser, STEREO/SECCHI Stereoscopic Observations Constraining the Initiation of Polar Coronal Jets, The Astrophysical Journal Letters, 680, 73-76, 2008.

Pesnell, W. D., B. J. Thompson, and P. C. Chamberlin, The Solar Dynamics Observatory (SDO), Solar Phys., 275, 3-15, doi:10.1007/ s11207-011-9841-3, 2012.

Savcheva, A., J. Cirtain, E. E. DeLuca, L. L. Lundquist, L. Golub, M. Weber, M. Shimojo, K. Shibasaki, T. Sakao, N. Narukage, et al., A study of polar jet parameters based on hinode xrt observations, PASJ, 59(sp3), S771-S778, 2007

Schmieder, B., G. Simnett, E. Tandberg-Hanssen, and P. Mein, An Example of The Association of X-Ray and UV Emission with $\mathrm{H} \alpha$ Surges, $A \& A$, 201, 327-338, 1988.

Shibata, K., and Y. Uchida, A Magnetodynamic Mechanism for the Formation of Astrophysical Jets. I. Dynamical Effects of the Relaxation of Nonlinear Magnetic Twists, PASJ, 37, 31-46, 1985

Shibata, K., M. Shimojo, T. Yokoyama, and M. Ohyama, Theory and Observations of X-Ray Jets., in Astronomical Society of the Pacific Conference Series, Astronomical Society of the Pacific Conference Series, vol. 111, pp. 29-38, 1996.

Shibata, K., T. Nakamura, T. Matsumoto, K. Otsuji, T. J. Okamoto, N. Nishizuka, T. Kawate, H. Watanabe, S. Nagata, S. UeNo, R. Kitai, S. Nozawa, S. Tsuneta, Y. Suematsu, K. Ichimoto, T. Shimizu, Y. Katsukawa, T. D. Tarbell, T. E. Berger, B. W. Lites, R. A. Shine, and A. M. Title, Chromospheric Anemone Jets as Evidence of Ubiquitous Reconnection, Science, 318, 1591-, doi:10.1126/science.1146708, 2007.

Shimojo, M., N. Narukage, R. Kano, T. Sakao, S. Tsuneta, K. Shibasaki, J. W. CIrtain, L. L. LUndquist, K. K. REeves, and A. SAvcheva, Fine Structures of Solar X-Ray Jets Observed with the X-Ray Telescope aboard Hinode, PASJ, 59, S745-S750, 2007.

Tian, H., S. W. McIntosh, S. R. Habbal, and J. He, Observation of Highspeed Outflow on Plume-like Structures of the Quiet Sun and Coronal Holes with Solar Dynamics Observatory/Atmospheric Imaging Assembly, ApJ, 736, 130, doi:10.1088/0004-637X/736/2/130, 2011.

Tian, H., E. E. DeLuca, S. R. Cranmer, B. De Pontieu, H. Peter, J. MartinezSykora, L. Golub, S. McKillop, K. K. Reeves, M. P. Miralles, P. McCauley, S. Saar, P. Testa, M. Weber, N. Murphy, J. Lemen, a. Title, P. Boerner, N. Hurlburt, T. D. Tarbell, J. P. Wuelser, L. Kleint, C. Kankelborg, S. Jaeggli, M. Carlsson, V. Hansteen, and S. W. McIntosh, Prevalence of small-scale jets from the networks of the solar transition region and chromosphere, Science, 346(6207), 1255,711-1255,711, doi: 10.1126/science.1255711, 2014.

Tsiropoula, G., and K. Tziotziou, The role of chromospheric mottles in the mass balance and heating of the solar atmosphere, $A \& A, 424,279-288$, doi:10.1051/0004-6361:20035794, 2004.

Xu, A., D. Ping, and S. Yin, The Rotation Mass Motion in Solar Surge, ACTA Astronomica Sinica, 25, 119-126, 1984. 